\title{
Tendencia de la mortalidad por enfermedades intersticiales en México, período 2000-2010
}

\author{
David Martínez-Briseño, Cecilia García-Sancho, $\bowtie$ Rosario Fernández-Plata, \\ Francisco Franco-Marina, Luis Torre-Bouscuolet, José Rogelio Pérez-Padilla
}

Instituto Nacional de Enfermedades Respiratorias Ismael Cosío Villegas, Ciudad de México.

Trabajo recibido: 19-VIII-2014; aceptado: 26-IX-2014

\begin{abstract}
RESUMEN. Antecedentes: Son escasos los estudios epidemiológicos que analizan las causas de muerte por enfermedades pulmonares intersticiales desde el punto de vista poblacional. Objetivo: Analizar la tendencia de la tasa de mortalidad estandarizada por edad, y las muertes anuales específicas por grupo de edad y sexo por enfermedades pulmonares intersticiales en México durante el período $2000-2010$. Métodos: A partir de los datos oficiales del número de muertes por causa (Instituto Nacional de Estadística y Geografía) y de los datos de población de la CONAPO, se calculó la tasa de mortalidad estandarizada por edad para hombres y para mujeres mediante el método directo. También fueron calculadas las tasas de mortalidad por enfermedades intersticiales en dos grupos de edad, de 45-64 años y de 65 y más años para cada uno de los sexos. Los grupos de la Clasificación Internacional de las Enfermedades analizados fueron J60-J80, J82-J84 y J99.1. Resultados: Se registraron 22,600 defunciones codificadas por enfermedades intersticiales pulmonares del total de $5,420,059$ muertes ocurridas durante el período de 2000-2010 (0.4\%). El código CIE-10 con mayor porcentaje de muertes fue el J84 que correspondió a «otras enfermedades pulmonares intersticiales» con el $80.2 \%$ de las muertes durante el período $(18,127 / 22,600)$. Este grupo incluye a las enfermedades reumáticas y autoinmunes que presentan involucro pulmonar. En la mortalidad estandarizada por edad en ambos sexos, se observó un incremento sostenido en la mortalidad por enfermedades intersticiales pulmonares durante todo el período; sin embargo, los hombres presentaron tasas más altas. La descripción de las tasas anuales de mortalidad específicas por grupo de edad y sexo mostraron un incremento sostenido de las tasas durante todo el período en ambos sexos en la población de 65 y más años. Conclusión: Se muestra la relevancia de las enfermedades intersticiales como causa de muerte en México. En los próximos años se requerirán recursos adicionales para la atención de estas enfermedades.
\end{abstract}

Palabras clave: Enfermedades intersticiales, México, mortalidad.

ABSTRACT. Background: Worldwide almost no epidemiologic data are available on the mortality of interstitial lung diseases in the general population. Its epidemiology in Mexico has not been well characterized. Objectives: We aimed to analyze the trends in mortality age-standardized by interstitial lung diseases from 2000 to 2010 in Mexico and the annual death rates by age and sex. Methods: The official National Database of Mortality was used to establish date and cause of deaths (Instituto Nacional de Estadística y Geografía [INEGI] from 2000 to 2010 in Mexico. Age-standardized rates were calculated by direct method; annual rates of death by sex in two age-groups (45-64 years old and 65 or more years old) were calculated. The codes of the International Statistical Classification of Diseases and Related Health Problems 10th Revision (CIE-10) J60-J80, J82-J84 y J99.1 were analyzed. Results: Of 5,420,059 deaths registered, 22,600 were diagnosed as death of an interstitial lung disease between 2000 and 2010 (0.4\%). The CIE-10 code with a higher percentage of deaths was J84: «Other pulmonary interstitial diseases» with $80.2 \%(18,127 / 22,600)$. This code includes the rheumatic diseases and autoimmune diseases that exhibit a lung interstitial disease. The age-standardized mortality rates by lung interstitial diseases shown an increasing trend during 2000-2010, but men exhibited higher rates than women; the description of annual mortality shown that the rates specific by age and sex were increasing in both sex but higher in male than female. The age group more affected was of 65 or more years old. Conclusions: This article shows the relevance of interstitial lung diseases as a death cause in Mexico. In the next years will be required additional resources to medical care in these diseases.

Key words: Interstitial lung disease, Mexico, mortality.

\section{INTRODUCCIÓN}

Son escasos los estudios epidemiológicos que describen el comportamiento de las enfermedades intersticiales a nivel poblacional, tanto en México como en otros países. Éste es un grupo de enfermedades respiratorias de causa desconocida y otras secundarias a diversas exposiciones ocupacionales tales como: exposición a carbón, asbesto, sílice, otros polvos inorgánicos, polvos orgánicos y a diferentes humos, vapores y sustancias 
químicas o a varias enfermedades autoinmunes, como las artritis, artropatías, lupus eritematoso diseminado, dermatopolimiositis y esclerosis sistémica, entre otras enfermedades.

El análisis de la mortalidad por este tipo de enfermedades permite identificar su frecuencia, así como los requerimientos más apremiantes en recursos para la salud y entrenamiento actuales y futuros y posibles puntos débiles en prevención y asistencia. En este contexto, el objetivo del presente estudio fue analizar la tendencia de la tasa de mortalidad estandarizada por edad y las muertes anuales específicas por grupo de edad y sexo por enfermedades pulmonares intersticiales en México durante el período 2000-2010.

\section{MATERIAL Y MÉTODOS}

A partir de los datos oficiales del número de muertes por causa (Instituto Nacional de Estadística y Geografía [INEGI] $]^{1}$ y de los datos de población de la CONAPO, se calculó la tasa de mortalidad estandarizada por edad para hombres y para mujeres mediante el método directo. Adicionalmente se calcularon las tasas de muertes anuales por enfermedades intersticiales en dos grupos de edad, de 45-64 años y de 65 y más años para cada uno de los sexos. Para las tasas específicas por edad y sexo, el numerador fue el número de muertes en cada uno de los siguientes grupos de la Clasificación Internacional de las Enfermedades, décima edición: J60-J80, J82-J84 y J99.1 y el denominador la población específica por edad y sexo. ${ }^{2}$ La descripción de los códigos analizados en este estudio se presenta en la tabla 1.

\section{RESULTADOS}

Se registraron 22,600 defunciones codificadas por enfermedades intersticiales pulmonares del total de $5,420,059$ muertes ocurridas durante el período de 2000-2010 (0.4\%). El código ClE-10 con mayor porcentaje de muertes fue el J84 que correspondió a «otras enfermedades pulmonares intersticiales» con el $80.2 \%$ de las muertes durante el período $(18,127 / 22,600)$. En este grupo se incluyen las muertes por enfermedades autoinmunes del tejido conectivo con afectación pulmonar (tabla 1). El resto de las muertes debido a enfermedades intersticiales, agrupadas en los rubros $\mathrm{J} 60$ a J80 de la CIE-10, son las causadas por agentes externos, casi todas ellas clasificadas como neumoconiosis y comprenden el $20 \%$ del total de muertes por enfermedades intersticiales.

En la mortalidad estandarizada por edad hubo un incremento sostenido en la mortalidad por enfermedades intersticiales pulmonares durante todo el período;
Tabla 1. Descripción de los códigos de la CIE-10 analizados en este estudio.

\begin{tabular}{|c|c|}
\hline CIE-10 & Descripción \\
\hline J60 & Neumoconiosis de los mineros del carbón \\
\hline J61 & $\begin{array}{l}\text { Neumoconiosis debida al asbesto y a otras fibras } \\
\text { minerales }\end{array}$ \\
\hline J62 & Neumoconiosis debida a polvo de sílice \\
\hline J63 & Neumoconiosis debida a otros polvos inorgánicos \\
\hline J64 & Neumoconiosis, no especificada \\
\hline J65 & Neumoconiosis asociada con tuberculosis \\
\hline J66 & $\begin{array}{l}\text { Enfermedades de las vías aéreas debidas a polvos } \\
\text { orgánicos específicos }\end{array}$ \\
\hline J67 & $\begin{array}{l}\text { Neumonitis debida a hipersensibilidad al polvo } \\
\text { orgánico }\end{array}$ \\
\hline J68 & $\begin{array}{l}\text { Afecciones respiratorias debidas a inhalación de } \\
\text { gases humos, vapores y sustancias químicas }\end{array}$ \\
\hline J69 & Neumonitis debida a sólidos y líquidos \\
\hline $\mathrm{J} 70$ & $\begin{array}{l}\text { Afecciones respiratorias debidas a otros agentes } \\
\text { externos }\end{array}$ \\
\hline J80 & Síndrome de dificultad respiratoria del adulto \\
\hline J82 & Eosinofilia pulmonar, no clasificada en otra parte \\
\hline J84 & Otras enfermedades pulmonares intersticiales \\
\hline J99.1 & $\begin{array}{l}\text { Trastornos respiratorios en enfermedades clasifica- } \\
\text { das en otra parte }\end{array}$ \\
\hline
\end{tabular}

se observó un incremento mayor en hombres, de 8 defunciones por 100,000 hombres (2000) a 13 muertes por 100,000 hombres (2010). En las mujeres también hubo un ascenso de 6 defunciones por 100,000 mujeres (2000) a 10 muertes por 100,000 mujeres. A pesar de existir una brecha durante todo el período entre la mortalidad estandarizada por edad entre hombres y mujeres, esta diferencia entre ambos sexos no fue estadísticamente significativa (figura 1). La descripción de las tasas específicas por grupo de edad y sexo mostró en las mujeres de 65 y más años un incremento sostenido en las tasas de muertes anuales, de 18 muertes por 100,000 mujeres (2000) a 30 defunciones por 100,000 mujeres (2010) ( $p=0.08)$. Por su parte, también en los hombres de 65 y más años existió un incremento sostenido en las tasas anuales de mortalidad, de 22 defunciones por 100,000 hombres (2000) a 40 muertes por 100,000 hombres (2010) $(p=0.02)$ (figura 2).

\section{DISCUSIÓN}

Los resultados principales de este estudio son: a) la tendencia en la tasa de mortalidad por enfermedades pulmonares intersticiales tiene una tendencia al incre- 
mento durante el período de 2000 a 2010; b) los hombres presentan tasas de mortalidad significativamente más altas durante todo el período, en comparación con las mujeres.
Se ha descrito que existe un subdiagnóstico de las enfermedades pulmonares intersticiales. El incremento en las tasas de mortalidad observado en nuestro estudio podría ser explicado por una mejora paulatina

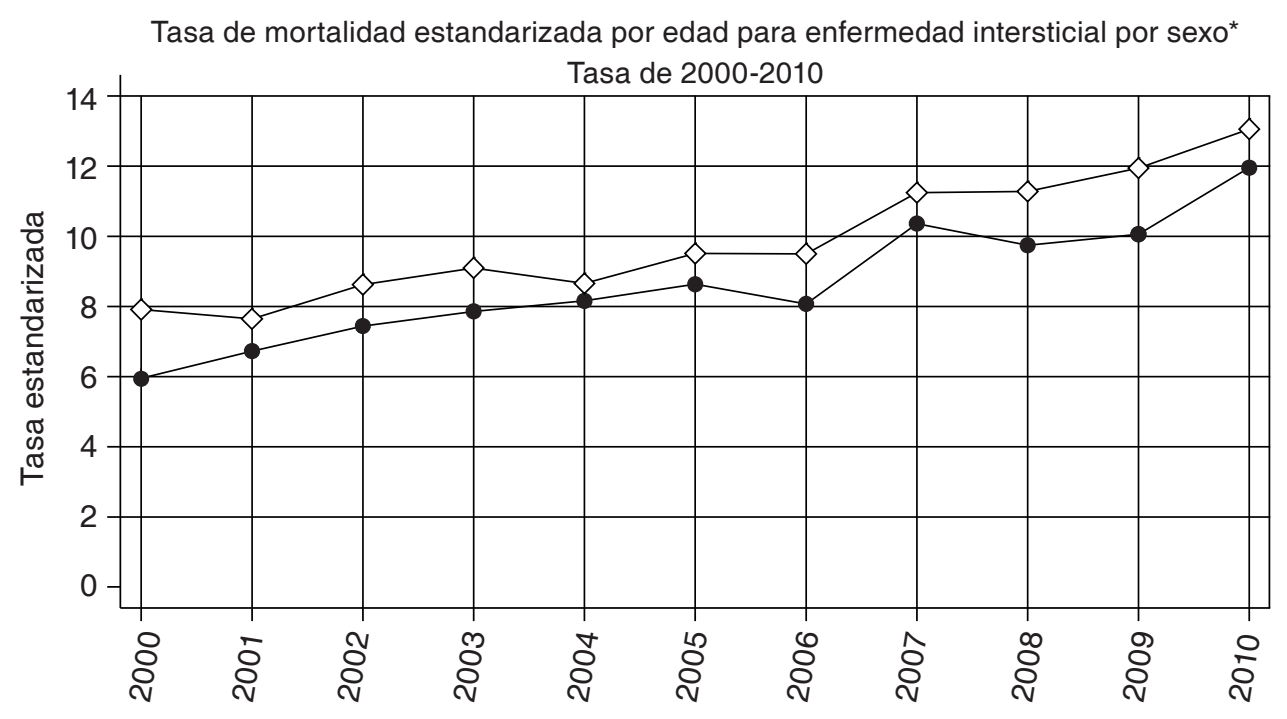

Año de defunción

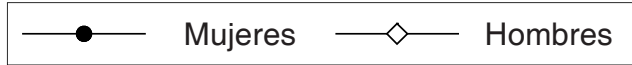

* Tasas por cada 100,000 habitantes

Muertes anuales por grupo de edad y sexo por enfermedad intersticial

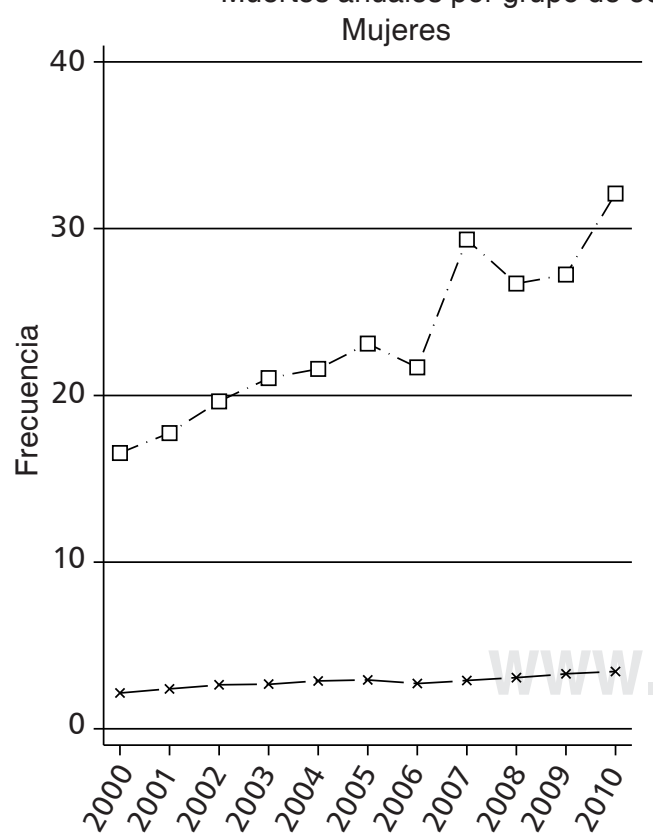

Año de defunción

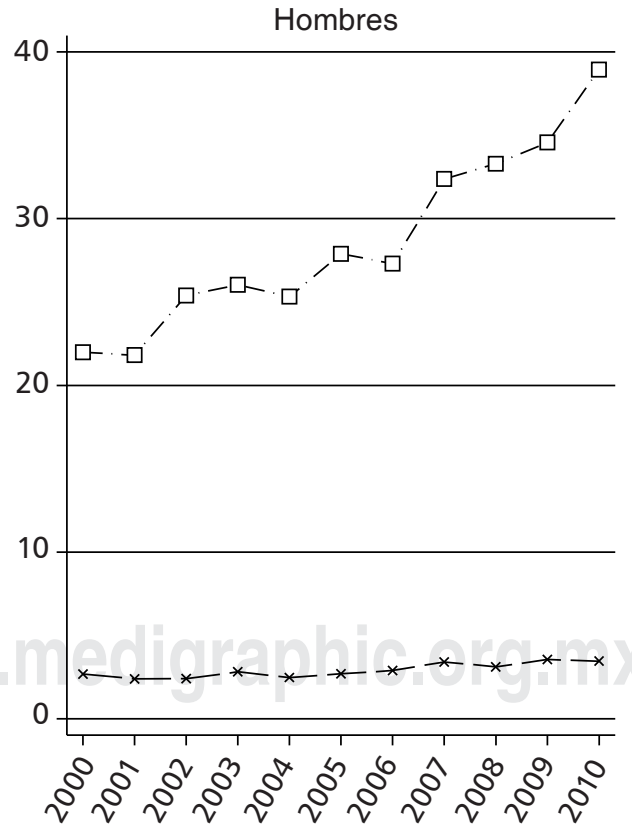

$-× 46-64-\square \cdot-65+$
Año de defunción $-\nsim 46-64-\cdot \cdot-65+$
Figura 1.

Tasa de mortalidad estandarizada para las mujeres (círculo negro) y para los hombres (rombo vacío).

Figura 2.

Muertes anuales para el grupo de edad de 46 a 64 años (equis) y de 65 años o más (cuadrado vacío). 
en el diagnóstico y notificación, ya que se trata de enfermedades raras con diagnóstico más bien sofisticado disponible en pocos sitios. ${ }^{3}$ Desconocemos la frecuencia de subdiagnóstico o subcertificación de enfermedades intersticiales en México, pero sin duda debe existir y podría estar reduciéndose conforme pasa el tiempo. Sin embargo, es más probable que dicho incremento sea un fenómeno real pues se ha visto en forma similar en otros países desarrollados donde en principio no tendrían tanto problema de subdiagnóstico en reducción.

Estudios realizados en otros países muestran la magnitud del subdiagnóstico que existe en las enfermedades pulmonares intersticiales. En 134 pacientes fallecidos por una enfermedad pulmonar intersticial en Nuevo México, sólo en $46 \%$ de los pacientes aparecía este diagnóstico en alguna parte del certificado de defunción y sólo en $15 \%$ como causa inmediata de la muerte. Para los pacientes con alguna enfermedad pulmonar intersticial mencionada en cualquier parte del certificado de defunción, la concordancia entre el diagnóstico antes de la muerte y aquellos diagnósticos registrados en el certificado de defunción fue de $76 \%$. Los datos del estado de Nuevo México mostraron que en el certificado de defunción, se asignó un diagnóstico de enfermedad intersticial pulmonar como causa de muerte en sólo $22 \%$ de los pacientes. La concordancia entre el diagnóstico hecho antes de la muerte y los diagnósticos registrados en los datos de mortalidad estatales fue nada más de $21 \%{ }^{4}$ Otro estudio recién realizado en la India mostró que la sarcoidosis, la fibrosis pulmonar idiopática y la neumonía intersticial no específica, fueron las enfermedades pulmonares intersticiales más comunes en el Norte de la India. Las enfermedades intersticiales se diagnosticaron frecuente y erróneamente como tuberculosis. Esto se debe a la carencia de las facilidades diagnósticas que requiere el diagnóstico temprano de las enfermedades intersticiales en los países en desarrollo. ${ }^{5}$

La prevalencia de enfermedades pulmonares intersticiales en pacientes con enfermedades autoinmunes $\mathrm{y} / \mathrm{o}$ reumáticas es muy variable, dependiendo de las fuentes de datos que se consulten. En países con sistemas de información nacionales y con encuestas de base poblacional, la prevalencia de enfermedad pulmonar intersticial en pacientes con esclerosis sistémica varió de 19 a 47\%, dependiendo de la definición utilizada. ${ }^{6}$ Para Estados Unidos la prevalencia de fibrosis pulmonar idiopática, enfermedad pulmonar intersticial difusa crónica de causa desconocida, se estimó en 14.0 por 100,000 habitantes y la incidencia en 6.8 por 100,000 habitantes para el período de enero de 1996 a diciembre del año 2000. ${ }^{7}$ Estos estudios no son comparables con el nuestro, ya que no describen la mortalidad asociada a ambas enfermedades. En un tercer estudio de base poblacional realizado en Bélgica, en 237 pacientes registrados, los diagnósticos más frecuentes fueron: sarcoidosis, 27\%; fibrosis pulmonar idiopática, $20 \%$; neumonitis por hipersensibilidad, 14\%; enfermedad vascular y del tejido conjuntivo, $10 \%$ (de los cuales $54 \%$ ocurrieron en pacientes con artritis reumatoide). ${ }^{8}$

Se ha descrito que la prevalencia de enfermedad intersticial pulmonar en los pacientes con artritis reumatoide es de aproximadamente $5 \% .{ }^{9}$ En estudios de series de casos, en 81 pacientes con diferentes enfermedades del tejido conectivo, $85.1 \%$ mostraron datos de enfermedad pulmonar intersticial en la tomografía. ${ }^{10}$ En el caso de la polimiositis/dermatomiositis, la enfermedad intersticial pulmonar no específica parece ser la forma histológica más común observada en estos pacientes, de 40 a $80 \% .{ }^{11}$ Un estudio realizado en la India que incluyó 140 pacientes con artritis reumatoide, la manifestación extraarticular más frecuente fue la enfermedad intersticial pulmonar con $9.3 \% .^{12}$ En contraste, en España, en 788 pacientes con artritis reumatoide, la prevalencia de enfermedad intersticial pulmonar fue de $3.7 \%(2.4$ a $5.0 \%) .{ }^{13}$ En pacientes con dermatomiositis y polimiositis, la frecuencia de enfermedad intersticial pulmonar fue de $39 \%$ de un total de 50 pacientes. ${ }^{14}$ En 28 pacientes con espondilitis anquilosante temprana, el $64.3 \%$ mostraron alteraciones pulmonares en la tomografía computarizada. En esta serie de casos de espondilitis anquilosante temprana, la afectación de vías aéreas pequeñas fue tan frecuente como la enfermedad intersticial pulmonar..$^{15}$ Estos estudios permiten concluir que las enfermedades intersticiales pulmonares en pacientes con enfermedades del tejido conectivo son frecuentes y que son la principal causa de muerte por enfermedades intersticiales, en comparación con las muertes debidas a exposiciones laborales.

En nuestro estudio, el grupo de edad más afectado, tanto en hombres como en mujeres fue el de 65 y más años. Estos datos son consistentes con los que refieren una asociación entre el envejecimiento y un incremento en la incidencia de enfermedades pulmonares intersticiales. Esto se debe a que las enfermedades intersticiales son mayoritariamente de enfermedades asociadas a edad avanzada, por lo que las tasas crudas de mortalidad por estas enfermedades se afectan por el envejecimiento poblacional, presente en México $y$ en todo el mundo. El envejecimiento se asocia a múltiples enfermedades crónicas, incluyendo a las enfermedades respiratorias. La edad avanzada se asocia con un mayor riesgo de algunas formas de enfermedad pulmonar intersticial, y este riesgo se refleja especialmente por el aumento significativo de la inci- 
dencia de fibrosis pulmonar idiopática en la vejez. ${ }^{16,17}$ Se considera que la fibrosis pulmonar idiopática es la enfermedad del envejecimiento más frecuente de las enfermedades pulmonares intersticiales y la mayoría de casos se presenta en sujetos de más de 70 años. Se considera también como una enfermedad producto del tabaquismo. ${ }^{18}$ Nuestros resultados también muestran que existe un incremento con la edad en la tasa de mortalidad por grupo de edad y sexo, siendo el más afectado el grupo de 65 y más años. Las tasas de prevalencia e incidencia de enfermedades pulmonares intersticiales más altas en hombres que en mujeres se ha descrito en otros estudios epidemiológicos. Esta sobremortalidad masculina puede explicarse por las múltiples exposiciones que los hombres tienen en el lugar de trabajo. ${ }^{19}$

Existen varios factores de riesgo para las enfermedades pulmonares intersticiales que pueden explicar el aumento de la mortalidad. El incremento en las tasas de mortalidad por enfermedades pulmonares intersticiales podría deberse a exposiciones acumulativas de tabaquismo activo y pasivo, ${ }^{20,21}$ a factores de riesgo laborales, ${ }^{22}$ factores exposicionales adicionales, ${ }^{23} \mathrm{y}$ otros no bien identificados mucho más probablemente que a un deterioro en la atención de los pacientes. En México, en pacientes derechohabientes del Instituto Mexicano del Seguro Social, durante el período de 1994 a 2004 se observó una reducción del número de casos de neumoconiosis, pero aún sigue existiendo un retraso en el diagnóstico y debido a esto los trabajadores afectados tienen, al momento del diagnóstico, secuelas irreversibles. ${ }^{24}$

En las enfermedades intersticiales, la referencia tardía a un centro especializado impacta negativamente la supervivencia de los pacientes. El retraso en el acceso a un centro de atención de tercer nivel está asociado a una tasa muy alta de mortalidad por fibrosis pulmonar idiopática, independientemente de la gravedad de la enfermedad. ${ }^{25}$

Nuestro grupo mostró en un estudio de casos y controles retrospectivo de base hospitalaria que la diabetes tipo 2 estaba asociada a la presencia de fibrosis pulmonar idiopática. ${ }^{26}$ Sin embargo, esta asociación no se pudo comprobar en un estudio de casos y controles prospectivo con controles poblacionales; en este estudio el factor de riesgo de fibrosis pulmonar idiopática más importante fue la agregación familiar de la enfermedad. ${ }^{27}$ Un factor de riesgo adicional que se asocia a esta enfermedad es el tabaquismo. En México, la adicción al tabaquismo es alta de $21.7 \%$ en el 2011. ${ }^{28}$ El humo de leña presenta una asociación débil con el desarrollo de enfermedades intersticiales pulmonares. ${ }^{29}$

\section{Ventajas y limitaciones del estudio}

Una ventaja importante en este estudio fue el incluir a todas las enfermedades intersticiales que tenían esta clasificación en el certificado de defunción. El código J84 incluye específicamente a las «otras enfermedades pulmonares intersticiales» que representó el $80.2 \%$ de las muertes analizadas. El uso de estos rubros de la CIE-10 permitió hacer un estudio epidemiológico de estas enfermedades, y las tasas y tendencias que se describen se presentan en México por primera vez.

Nuestro grupo de trabajo hizo el análisis de la mortalidad de acuerdo a la clasificación de la causa básica de muerte tal y como lo proporciona el INEGI y no un análisis por causa múltiple del certificado de defunción, lo cual puede subestimar la mortalidad debida a enfermedades intersticiales.

Los resultados de nuestro estudio muestran la relevancia de las enfermedades respiratorias intersticiales como causa de muerte, así como un incremento en la mortalidad por esas mismas enfermedades. Este aumento puede estar asociado con el envejecimiento de la población ya que el grupo más afectado por estas enfermedades es el de 65 y más años. En los próximos años se requerirán recursos adicionales para diagnosticar y tratar las enfermedades intersticiales, así como una mejor preparación del personal de salud, sobre todo en los centros de referencia para enfermedades respiratorias.

\section{REFERENCIAS}

1. Instituto Nacional de Estadística y Geografía. Mortalidad, defunciones generales por grupos de edad y sexo, 1990 a 2010. Consultado el 15 de agosto de 2013.

2. World Health Organization. International Statistical Classification of Diseases and Related Health Problems 10th Revision. Access date: August 15, 2013. Available from: http://apps.who.int/classifications/icd10/browse/2010/

3. Wells AU. The revised ATS/ERS/JRS/ALAT diagnostic criteria for idiopathic pulmonary fibrosis (IPF)--practical implications. Respir Res 2013;14(Suppl 1):S2. doi: 10.1186/1465-9921-14-S1-S2.

4. Coultas DB, Hughes MP. Accuracy of mortality data for interstitial lung diseases in New Mexico, USA. Thorax 1996;51(7):717-720.

5. Kumar R, Gupta N, Goel N. Spectrum of interstitial lung disease at a tertiary care centre in India. Pneumonol Alergol Pol 2014;82(3):218-226. doi: 10.5603/ PiAP.2014.0029.

6. Vonk MC, Broers B, Heijdra YF, et al. Systemic sclerosis and its pulmonary complications in The Netherlands: an epidemiological study. Ann Rheum Dis 2009;68(6):961965. doi: 10.1136/ard.2008.091710.

7. Raghu G, Weycker D, Edelsberg J, Bradford WZ, Oster $\mathrm{G}$. Incidence and prevalence of idiopathic pulmonary fibrosis. Am J Respir Crit Care Med 2006;174(7):810-816. 
8. Roelandt M, Demedts M, Callebaut W, et al. Epidemiology of interstitial lung disease (ILD) in Flanders: registration by pneumologists in 1992-1994. Working group on ILD, VRGT. Vereniging voor Respiratoire Gezondheidszorg en Tuberculosebestrijding. Acta Clin Belg 1995;50(5):260-268.

9. Kelly CA, Saravanan V, Nisar M, et al; British Rheumatoid Interstitial Lung (BRILL) Network. Rheumatoid arthritis-related interstitial lung disease: associations, prognostic factors and physiological and radiological characteristics-a large multicentre UK study. Rheumatology (Oxford) 2014;53(9):1676-1682. doi: 10.1093/ rheumatology/keu165.

10. Afeltra A, Zennaro D, Garzia P, et al. Prevalence of interstitial lung involvement in patients with connective tissue diseases assessed with high-resolution computed tomography. Scand J Rheumatol 2006;35(5):388-394.

11. Marie I, Dominique S. Pulmonary damage during polymyositis and dermatomyositis: interstitial lung disease. Presse Med 2006;35(4 Pt 2):683-695.

12. Bharadwaj A, Haroon N. Interstitial lung disease and neuropathy as predominant extra-articular manifestations in patients with rheumatoid arthritis: a prospective study. Med Sci Monit 2005;11(10):CR498-502.

13. Carmona L, González-Alvaro I, Balsa A, Angel-Belmonte M, Tena X, Sanmartí R. Rheumatoid arthritis in Spain: occurrence of extra-articular manifestations and estimates of disease severity. Ann Rheum Dis 2003;62(9):897-900.

14. Selva-O'Callaghan A, Labrador-Horrillo M, MuñozGall X, et al. Polymyositis/dermatomyositis-associated lung disease: analysis of a series of 81 patients. Lupus 2005;14(7):534-542.

15. Kiris A, Ozgocmen S, Kocakoc E, Ardicoglu O, Ogur E. Lung findings on high resolution CT in early ankylosing spondylitis. Eur J Radiol 2003;47(1):71-76.

16. Meyer KC. Interstitial lung disease in the elderly: pathogenesis, diagnosis and management. Sarcoidosis Vasc Diffuse Lung Dis 2011;28(1):3-17.

17. Selman M, Rojas M, Mora AL, Pardo A. Aging and interstitial lung diseases: unraveling an old forgotten player in the pathogenesis of lung fibrosis. Semin Respir Crit Care Med 2010;31(5):607-617. doi: 10.1055/s-0030-1265901.

18. Cordier JF, Cottin V. Neglected evidence in idiopathic pulmonary fibrosis: from history to earlier diagnosis. Eur Respir J 2013;42(4):916-923. doi: 10.1183/09031936.00027913.

19. Coultas DB, Zumwalt RE, Black WC, Sobonya RE. The epidemiology of interstitial lung diseases. Am J Respir Crit Care Med 1994;150(4):967-972.

20. Perez-Padilla R, Schilmann A, Riojas-Rodriguez H. Respiratory health effects of indoor air pollution. Int J Tuberc Lung Dis 2010;14(9):1079-1086.

21. Baumgartner KB, Samet JM, Stidley CA, Colby TV, Waldron JA. Cigarette smoking: a risk factor for idio- pathic pulmonary fibrosis. Am J Respir Crit Care Med 1997;155(1):242-248.

22. Lido AV, Kitamura S, Oliveira JI, Lucca SR, Azevedo VA, Bagatin E. Occupational exposure and occurrence of pneumoconioses in Campinas, Brazil, 1978-2003. J Bras Pneumol 2008;34(6):367-372.

23. Baumgartner KB, Samet JM, Coultas DB, et al. Occupational and environmental risk factors for idiopathic pulmonary fibrosis: a multicenter case-control study. Collaborating Centers. Am J Epidemiol 2000;152(4):307315.

24. López-Rojas $P$, Nava-Larraguivel R, Salinas-Tovar S, Santos-Celis R, Marín-Cotoñieto IA, Méndez-Vargas MM. Pneumoconiosis in affiliated workers have irreversible sequelae. Rev Med Inst Mex Seguro Soc 2008;46(2):163170.

25. Lamas DJ, Kawut SM, Bagiella E, Philip N, Arcasoy SM, Lederer DJ. Delayed access and survival in idiopathic pulmonary fibrosis: a cohort study. Am J Respir Crit Care Med 2011;184(7):842-847. doi: 10.1164/rccm.201104-06680C.

26. García-Sancho Figueroa MC, Carrillo G, Pérez-Padilla R, et al. Risk factors for idiopathic pulmonary fibrosis in a Mexican population. A case-control study. Respir Med 2010;104(2):305-309. doi: 10.1016/j.rmed.2009.08.013.

27. García-Sancho C, Buendía-Roldán I, Fernández-Plata $\mathrm{MR}$, et al. Familial pulmonary fibrosis is the strongest risk factor for idiopathic pulmonary fibrosis. Respir Med 2011;105(12):1902-1907. doi: 10.1016/j. rmed.2011.08.022

28. Instituto Nacional de Psiquiatría Ramón de la Fuente Muñiz; Instituto Nacional de Salud Pública; Secretaría de Salud. Encuesta Nacional de Adicciones 2011: Reporte de tabaco. Reynales-Shigematsu LM, Guerrero-López CM, Lazcano-Ponce E, et al. México, D.F., México: INPRFM, 2012. Disponible en: www.inprf.gob.mx, www.conadic. gob.mx, www.cenadic.salud.gob.mx, www.insp.mx).

29. Sood A. Indoor fuel exposure and the lung in both developing and developed countries: an update. Clin Chest Med 2012;33(4):649-665. doi: 10.1016/j. ccm.2012.08.003.

\author{
$\triangle$ Correspondencia: \\ Dra. en C. Cecilia García-Sancho, \\ Instituto Nacional de Enfermedades Respiratorias \\ Ismael Cosío Villegas. \\ Calzada de Tlalpan Núm. 4502, Colonia Sección \\ XVI, México, D.F., 14080. \\ Teléfono: 54871700 extensión 5238; \\ fax 56654623 \\ Correo electrónico: cegarsan@netscape.net; \\ cegarsanfi@gmail.com \\ Los autores declaran no tener conflicto de intereses
}

